\title{
The Bakry-Emery Ricci tensor and its applications to some compactness theorems
}

\section{Murat Limoncu}

Received: 6 December 2010 / Accepted: 21 March 2011 / Published online: 10 May 2011

(C) The Author(s) 2011. This article is published with open access at Springerlink.com

\begin{abstract}
Let $(M, g)$ be a complete and connected Riemannian manifold of dimension $n$. By using the Bakry-Emery Ricci curvature tensor on $M$, we prove two theorems which correspond to the Myers compactness theorem.
\end{abstract}

Keywords Laplacian of distance function · Index form · Diameter estimate

\section{Introduction}

The purpose of this article is to generalize the well-known Myers compactness theorem [5] by using the Bakry-Emery Ricci curvature (see [1,4] and [6]) on a complete and connected Riemannian manifold $(M, g)$ of dimension $n$.

In [6] (p. 380, Theorem 1.4), G. Wei and W. Wylie assumed that the Bakry-Emery Ricci curvature has a positive lower bound, i.e.,

$$
\operatorname{Ric}+\operatorname{Hess}(\phi) \geq(n-1) H>0
$$

and also assumed that $|\phi| \leq k$, where $\phi \in \mathcal{C}^{\infty}(M)$ is a smooth function. Under these assumptions, they proved that $M$ is compact and diameter has the upper bound

$$
\operatorname{diam}(M) \leq \frac{\pi}{\sqrt{H}}+\frac{4 k}{(n-1) \sqrt{H}} .
$$

In the following, we consider the same assumptions given by G. Wei and W. Wylie, but, for the diameter of $M$, we obtain a different upper bound which can be compared with (2):

Theorem 1 Let $(M, g)$ be a complete and connected Riemannian manifold of dimension $n$. If $(M, g)$ admits a smooth function $\phi \in \mathcal{C}^{\infty}(M)$ satisfying the inequalities

$$
\operatorname{Ric}+\operatorname{Hess}(\phi) \geq(n-1) H>0
$$

M. Limoncu $(\varangle)$

Department of Mathematics, Anadolu University, 26470 Eskişehir, Turkey

e-mail: mlimoncu@anadolu.edu.tr 
and $|\phi| \leq k$, then $M$ is compact and the diameter satisfies

$$
\operatorname{diam}(M) \leq \frac{\pi}{\sqrt{H}} \sqrt{1+\frac{2 \sqrt{2} k}{n-1}} .
$$

Comparing (2) and (4), we see that, when the positive constant $k$ satisfies

$$
k>\frac{(n-1) \pi}{8}(\sqrt{2} \pi-4),
$$

the upper bound (4) is sharper than the upper bound (2).

Instead of the assumption $|\phi| \leq k$ given in Theorem 1 , we can assume that $g(\nabla \phi, \nabla \phi) \in$ $\mathcal{C}^{\infty}(M)$ has an upper bound: In [2], Fernández-López and García-Río proved that, if $(M, g)$ admits a vector field $V$ satisfying the inequality Ric $+\mathcal{L}_{V} g \geq c>0$ where $\mathcal{L}_{V}$ denotes the Lie derivative, and $\sqrt{g(V, V)}$ has an upper bound, then $M$ is compact. However, no an upper bound to the diameter of $M$ is given in [2]. In [3], such a bound was obtained for the diameter of $M$. Namely, if we have the inequalities Ric $+\mathcal{L}_{V} g \geq(n-1) H>0$ and $\sqrt{g(V, V)} \leq \gamma$, then $M$ is compact and has

$$
\operatorname{diam}(M) \leq \frac{\pi}{(n-1) H}\left(\sqrt{2} \gamma+\sqrt{2 \gamma^{2}+(n-1)^{2} H}\right)
$$

(see [3]). When the vector field $V$ is taken to be $V=\frac{1}{2} \nabla \phi$, the above inequalities yield Ric $+\operatorname{Hess}(\phi) \geq(n-1) H>0$ and $g(\nabla \phi, \nabla \phi) \leq 4 \gamma^{2}$. The inequality (6) still holds and, under the $K=4 \gamma^{2}$, it can be also written as

$$
\operatorname{diam}(M) \leq \frac{\pi}{(n-1) H}\left(\sqrt{\frac{K}{2}}+\sqrt{\frac{K}{2}+(n-1)^{2} H}\right) .
$$

In the following Theorem $2, g(\nabla \phi, \nabla \phi) \in \mathcal{C}^{\infty}(M)$ has again an upper bound, but now it depends on both the positive constant $K$ and a distance function $r=d(., p)$ with respect to a fixed point $p \in M$. We obtain an upper bound for the diameter of $M$. It can be compared with the above bound (7):

Theorem 2 Let $(M, g)$ be a complete and connected Riemannian manifold of dimension $n$, and let $r$ be the distance function with respect to a fixed point $p \in M$, i.e., $r(x)=d(x, p)$. Suppose that $(M, g)$ admits a smooth function $\phi \in \mathcal{C}^{\infty}(M)$ such that

$$
(g(\nabla \phi, \nabla \phi))(x) \leq \frac{K}{r^{2}(x)}
$$

for all $x \in M-\{p\}$, where $K$ is a positive constant. If $(M, g)$ has the inequality

$$
\operatorname{Ric}+\operatorname{Hess}(\phi) \geq(n-1) H>0,
$$

then $M$ is compact and the diameter from $p$ satisfies

$$
\operatorname{diam}_{p}(M) \leq \sqrt{4 \sqrt{K}+n-1} \frac{\pi}{\sqrt{(n-1) H}} .
$$

In the Theorem 2, the diameter bound is given with respect to the point $p \in M$. In other words, the bound is for " $\operatorname{diam}_{p}(M)$ " not for " $\operatorname{diam}(M)$ ". But, by using the triangle inequality, we get

$$
\begin{aligned}
\operatorname{diam}(M) & =d\left(p^{\prime}, q^{\prime}\right) \leq d\left(p^{\prime}, p\right)+d\left(q^{\prime}, p\right) \\
& \leq \sqrt{4 \sqrt{K}+n-1} \frac{2 \pi}{\sqrt{(n-1) H}}
\end{aligned}
$$


where the distance between the points $p^{\prime}$ and $q^{\prime}\left(p^{\prime}, q^{\prime} \in M\right)$ gives the diameter of $M$. For the case $p \neq p^{\prime}$ and $p \neq q^{\prime}$, comparing (7) and (11), we see that, when the positive constant $\sqrt{K}$ satisfies

$$
\sqrt{K} \geq 16(n-1) H\left(1+\sqrt{1+\frac{3}{128 H}}\right),
$$

the upper bound (11) is sharper than the upper bound (7). If $p \in M$ directly gives the diameter of $M$, i.e., $p=p^{\prime}$ (or $p=q^{\prime}$ ), then we can compare the bounds (7) and (10). In this special case where $\operatorname{diam}_{p}(M)=\operatorname{diam}(M)$, we see that, when the positive constant $\sqrt{K}$ satisfies

$$
\sqrt{K} \geq 8(n-1) H
$$

the upper bound (10) is sharper than the upper bound (7).

In order to prove the Theorem 1, we use the index form I of a minimizing unit speed geodesic segment. To prove the Theorem 2, we establish a comparison estimate for a modified Laplacian operator.

\section{Proofs of the theorems}

The gradient, Hessian and Laplacian of any smooth function $f \in \mathcal{C}^{\infty}(M)$ are defined by $g(\nabla f, V)=V(f),(\operatorname{Hess}(f))(V, W)=g\left(\nabla_{V} \nabla f, W\right)$ and $\Delta f=\operatorname{tr}(\nabla \nabla f)$ for all vector field $V, W$, respectively. For a distance function $r(x)=d(x, p)$ where $p \in M$ is a fixed point, it is well-known that $r$ is only smooth on $M-\left(C_{p} \cup\{p\}\right)$ where $C_{p}$ denotes the cut locus of the point $p \in M$. In addition to this fact, we have $\nabla r=\partial_{r}$ in the adapted coordinates with respect to the $r$, and also have $g(\nabla r, \nabla r)=1$ where $r$ is smooth.

Proof of Theorem 1 Let $p, q \in M$ and let $\sigma$ be a minimizing unit speed geodesic segment from $p$ to $q$ of length $\ell$. Considering a parallel orthonormal frame $\left\{E_{1}=\dot{\sigma}, E_{2}, \ldots, E_{n}\right\}$ along $\sigma$ and a smooth function $f \in \mathcal{C}^{\infty}([0, \ell])$ such that $f(0)=f(\ell)=0$, we have

$$
\mathrm{I}\left(f E_{i}, f E_{i}\right)=\int_{0}^{\ell}\left(g\left(\dot{f} E_{i}, \dot{f} E_{i}\right)-g\left(R\left(f E_{i}, \dot{\sigma}\right) \dot{\sigma}, f E_{i}\right)\right) d t
$$

where I denotes the index form of $\sigma$. From (14), we obtain

$$
\sum_{i=2}^{n} \mathrm{I}\left(f E_{i}, f E_{i}\right)=\int_{0}^{\ell}\left((n-1) \dot{f}^{2}-f^{2} \operatorname{Ric}(\dot{\sigma}, \dot{\sigma})\right) d t
$$

by $g(R(\dot{\sigma}, \dot{\sigma}) \dot{\sigma}, \dot{\sigma})=0$. Using the assumption (3) given in Theorem 1 in the integral expression (15), we get 


$$
\begin{aligned}
\sum_{i=2}^{n} \mathrm{I}\left(f E_{i}, f E_{i}\right) & \leq \int_{0}^{\ell}\left((n-1)\left(\dot{f}^{2}-H f^{2}\right)+f^{2}(\operatorname{Hess}(\phi))(\dot{\sigma}, \dot{\sigma})\right) d t \\
& =\int_{0}^{\ell}\left((n-1)\left(\dot{f}^{2}-H f^{2}\right)+f^{2} g\left(\nabla_{\dot{\sigma}} \nabla \phi, \dot{\sigma}\right)\right) d t \\
& =\int_{0}^{\ell}\left((n-1)\left(\dot{f}^{2}-H f^{2}\right)+f^{2} \dot{\sigma}(g(\nabla \phi, \dot{\sigma}))\right) d t
\end{aligned}
$$

where we have used the parallelism of the metric tensor $g$ and $\nabla_{\dot{\sigma}} \dot{\sigma}=0$. In the expression (16), the term $f^{2} \dot{\sigma}(g(V, \dot{\sigma}))$ equals to

$$
f^{2} \dot{\sigma}(g(\nabla \phi, \dot{\sigma}))=f^{2} \frac{d}{d t}(g(\nabla \phi, \dot{\sigma})(\sigma(t))) .
$$

When $g(\nabla \phi, \dot{\sigma})(\sigma(t))$ is denoted by $g(\nabla \phi, \dot{\sigma})$ for short, the expression (17) can be written as

$$
f^{2} \dot{\sigma}(g(\nabla \phi, \dot{\sigma}))=-2 f \dot{f} g(\nabla \phi, \dot{\sigma})+\frac{d}{d t}\left(f^{2} g(\nabla \phi, \dot{\sigma})\right)
$$

Here we also have $g(\nabla \phi, \dot{\sigma})=\dot{\sigma}(\phi)=\frac{d}{d t} \phi(\sigma(t))\left(=\frac{d \phi}{d t}\right.$ for short). Thus, the equation (18) yields

$$
\begin{aligned}
f^{2} \dot{\sigma}(g(\nabla \phi, \dot{\sigma})) & =-2 f \dot{f} \frac{d \phi}{d t}+\frac{d}{d t}\left(f^{2} g(\nabla \phi, \dot{\sigma})\right) \\
& =2 \phi \frac{d}{d t}(f \dot{f})-2 \frac{d}{d t}(\phi f \dot{f})+\frac{d}{d t}\left(f^{2} g(\nabla \phi, \dot{\sigma})\right) .
\end{aligned}
$$

Integrating both sides of (19), we obtain

$$
\begin{aligned}
\int_{0}^{\ell} f^{2} \dot{\sigma}(g(\nabla \phi, \dot{\sigma})) d t & =\int_{0}^{\ell} 2 \phi \frac{d}{d t}(f \dot{f}) d t-\left.2(\phi f \dot{f})\right|_{0} ^{\ell}+\left.\left(f^{2} g(\nabla \phi, \dot{\sigma})\right)\right|_{0} ^{\ell} \\
& =2 \int_{0}^{\ell} \phi \frac{d}{d t}(f \dot{f}) d t
\end{aligned}
$$

because of $f(0)=f(\ell)=0$. Now if we take $P=\phi$ and $Q=\frac{d}{d t}(f \dot{f})$, then we have, from the Cauchy-Schwarz inequality,

$$
\begin{aligned}
\int_{0}^{\ell} P Q d t & \leq\left(\int_{0}^{\ell} P^{2} d t\right)^{1 / 2}\left(\int_{0}^{\ell} Q^{2} d t\right)^{1 / 2} \\
\int_{0}^{\ell} \phi \frac{d}{d t}(f \dot{f}) d t & \leq\left(\int_{0}^{\ell} \phi^{2} d t\right)^{1 / 2}\left(\int_{0}^{\ell}\left(\frac{d}{d t}(f \dot{f})\right)^{2} d t\right)^{1 / 2} .
\end{aligned}
$$


Using the assumption $|\phi| \leq k$ given in Theorem 1 in (22), we obtain

$$
\int_{0}^{\ell} \phi \frac{d}{d t}(f \dot{f}) d t \leq k \sqrt{\ell}\left(\int_{0}^{\ell}\left(\frac{d}{d t}(f \dot{f})\right)^{2} d t\right)^{1 / 2}
$$

Thus, by using (23), the equation (20) yields

$$
\int_{0}^{\ell} f^{2} \dot{\sigma}(g(\nabla \phi, \dot{\sigma})) d t \leq 2 k \sqrt{\ell}\left(\int_{0}^{\ell}\left(\frac{d}{d t}(f \dot{f})\right)^{2} d t\right)^{1 / 2} .
$$

By virtue of (24), the inequality (16) becomes

$$
\begin{aligned}
\sum_{i=2}^{n} \mathrm{I}\left(f E_{i}, f E_{i}\right) \leq & \int_{0}^{\ell}\left((n-1)\left(\dot{f}^{2}-H f^{2}\right)\right) d t \\
& +2 k \sqrt{\ell}\left(\int_{0}^{\ell}\left(\frac{d}{d t}(f \dot{f})\right)^{2} d t\right)^{1 / 2} .
\end{aligned}
$$

In (25), if the function $f$ is taken to be $f(t)=\sin \left(\frac{\pi}{\ell} t\right)$, then we get

$$
\begin{aligned}
\sum_{i=2}^{n} \mathrm{I}\left(f E_{i}, f E_{i}\right) \leq & (n-1) \int_{0}^{\ell}\left(\frac{\pi^{2}}{\ell^{2}} \cos ^{2}\left(\frac{\pi}{\ell} t\right)-H \sin ^{2}\left(\frac{\pi}{\ell} t\right)\right) d t \\
& +\frac{2 k \pi^{2}}{\ell \sqrt{\ell}}\left(\int_{0}^{\ell} \cos ^{2}\left(\frac{2 \pi}{\ell} t\right) d t\right)^{1 / 2},
\end{aligned}
$$

and consequently

$$
\sum_{i=2}^{n} \mathrm{I}\left(f E_{i}, f E_{i}\right) \leq-\frac{1}{2 \ell}\left((n-1) H \ell^{2}-2 \sqrt{2} k \pi^{2}-(n-1) \pi^{2}\right) .
$$

Here, if $(n-1) H \ell^{2}-2 \sqrt{2} k \pi^{2}-(n-1) \pi^{2}>0$, then one has

$$
\sum_{i=2}^{n} \mathrm{I}\left(f E_{i}, f E_{i}\right)=\mathrm{I}\left(f E_{2}, f E_{2}\right)+\mathrm{I}\left(f E_{3}, f E_{3}\right) \ldots \mathrm{I}\left(f E_{n}, f E_{n}\right)<0
$$

which implies $\mathrm{I}\left(f E_{m}, f E_{m}\right)<0$, for some $2 \leq m \leq n$, Namely, the index form $\mathrm{I}$ is not positive semi-definite. However, this result contradicts with $\sigma$ being minimizing geodesic. Hence, we must take

$$
(n-1) H \ell^{2}-2 \sqrt{2} k \pi^{2}-(n-1) \pi^{2} \leq 0 .
$$

This inequality gives

$$
\ell \leq \frac{\pi}{\sqrt{H}} \sqrt{1+\frac{2 \sqrt{2} k}{n-1}}
$$

Thus, we have proved Theorem 1. 
Proof of Theorem 2 To prove Theorem 2, we consider a modified Laplace operator $\widetilde{\Delta}$ defined by

$$
\widetilde{\Delta} f=\Delta f-g(\nabla \phi, \nabla f)+F(f),
$$

where $\phi \in \mathcal{C}^{\infty}(M)$ is given in Theorem 2 and $F$ is a real valued smooth function defined on a subset of real line, and $F(f)$ denotes $F \circ f$. In the equation (31), when $f$ is taken to be the distance function $r$ given in Theorem 2, we obtain, on $M-\left(C_{p} \cup\{p\}\right)$,

$$
\begin{aligned}
g(\nabla r, \nabla \widetilde{\Delta} r) & =g(\nabla r, \nabla \Delta r-\nabla g(\nabla \phi, \nabla r)+\nabla F(r)) \\
& =g(\nabla r, \nabla \Delta r)-g(\nabla r, \nabla g(\nabla \phi, \nabla r))+F^{\prime}(r) \\
& =g(\nabla r, \nabla \Delta r)-(\operatorname{Hess}(\phi))(\nabla r, \nabla r)+F^{\prime}(r)
\end{aligned}
$$

where $F^{\prime}(r)=\frac{d}{d r} F(r)$. On the other hand, we have the well-known inequality

$$
0 \geq \operatorname{Ric}(\nabla r, \nabla r)+\frac{1}{n-1}(\Delta r)^{2}+g(\nabla r, \nabla \Delta r)
$$

on $M-\left(C_{p} \cup\{p\}\right)$. From (32) and (33), we find

$$
\begin{aligned}
0 \geq \operatorname{Ric}(\nabla r, \nabla r)+(\operatorname{Hess}(\phi))(\nabla r, \nabla r)-F^{\prime}(r) \\
+\frac{1}{n-1}(\Delta r)^{2}+g(\nabla r, \nabla \widetilde{\Delta} r) .
\end{aligned}
$$

It is obvious that we have

$$
\Delta r=\widetilde{\Delta} r+g(\nabla \phi, \nabla r)-F(r)
$$

by (31). Inserting (35) into (34), we obtain

$$
\begin{aligned}
0 \geq & \operatorname{Ric}(\nabla r, \nabla r)+(\operatorname{Hess}(\phi))(\nabla r, \nabla r)-F^{\prime}(r) \\
& +\frac{1}{n-1}(\widetilde{\Delta} r+g(\nabla \phi, \nabla r)-F(r))^{2}+g(\nabla r, \nabla \widetilde{\Delta} r) .
\end{aligned}
$$

By virtue of the inequality $(a \mp b)^{2} \geq \frac{1}{\gamma+1} a^{2}-\frac{1}{\gamma} b^{2}$ for all real numbers $a, b$ and positive real number $\gamma>0$, we obtain

$$
\begin{aligned}
(\widetilde{\Delta} r+g(\nabla \phi, \nabla r)-F(r))^{2} \geq & \frac{1}{\gamma+1}(\widetilde{\Delta} r+g(\nabla \phi, \nabla r))^{2} \\
& -\frac{1}{\gamma}(F(r))^{2}
\end{aligned}
$$

Using the same inequality, for the term " $(\widetilde{\Delta} r+g(\nabla \phi, \nabla r))^{2}$ " in the above inequality, we get

$$
\begin{aligned}
(\widetilde{\Delta} r+g(\nabla \phi, \nabla r)-F(r))^{2} \geq & \frac{1}{(\gamma+1) \eta+\gamma+1}(\widetilde{\Delta} r)^{2}-\frac{1}{\gamma}(F(r))^{2} \\
& -\frac{1}{(\gamma+1) \eta}(g(\nabla \phi, \nabla r))^{2}
\end{aligned}
$$


for all $\gamma, \eta>0$. Inserting (38) into (36) and denoting $\alpha=(n-1) \gamma>0, \beta=(n-1)(\gamma+1) \eta>0$ we obtain, on $M-\left(C_{p} \cup\{p\}\right)$,

$$
\begin{aligned}
0 \geq & \operatorname{Ric}(\nabla r, \nabla r)+(\operatorname{Hess}(\phi))(\nabla r, \nabla r)+\frac{1}{\alpha+\beta+n-1}(\widetilde{\Delta} r)^{2} \\
& +g(\nabla r, \nabla \widetilde{\Delta} r)-F^{\prime}(r)-\frac{1}{\alpha}(F(r))^{2}-\frac{1}{\beta}(g(\nabla \phi, \nabla r))^{2} .
\end{aligned}
$$

From the Cauchy-Schwarz inequality, we have

$$
(g(\nabla \phi, \nabla r))^{2} \leq g(\nabla \phi, \nabla \phi) g(\nabla r, \nabla r)=g(\nabla \phi, \nabla \phi),
$$

which implies

$$
-\frac{1}{\beta}(g(\nabla \phi, \nabla r))^{2} \geq-\frac{1}{\beta} g(\nabla \phi, \nabla \phi) .
$$

Using (41) in (39), one has

$$
\begin{aligned}
0 \geq & \operatorname{Ric}(\nabla r, \nabla r)+(\operatorname{Hess}(\phi))(\nabla r, \nabla r)+\frac{1}{\alpha+\beta+n-1}(\widetilde{\Delta} r)^{2} \\
& +g(\nabla r, \nabla \widetilde{\Delta} r)-F^{\prime}(r)-\frac{1}{\alpha}(F(r))^{2}-\frac{1}{\beta} g(\nabla \phi, \nabla \phi) .
\end{aligned}
$$

From the assumption (8) given in Theorem 2, we obtain, on $M-\left(C_{p} \cup\{p\}\right)$,

$$
\begin{aligned}
0 \geq & \operatorname{Ric}(\nabla r, \nabla r)+(\operatorname{Hess}(\phi))(\nabla r, \nabla r)+\frac{1}{\alpha+\beta+n-1}(\widetilde{\Delta} r)^{2} \\
& +g(\nabla r, \nabla \widetilde{\Delta} r)-F^{\prime}(r)-\frac{1}{\alpha}(F(r))^{2}-\frac{K}{\beta r^{2}} .
\end{aligned}
$$

In the above expression, if we take $\beta=\frac{4 K}{\alpha}$ and $F(r)=\frac{\alpha}{2 r}$, then the inequality (43) yields

$$
\begin{aligned}
0 \geq & \operatorname{Ric}(\nabla r, \nabla r)+(\operatorname{Hess}(\phi))(\nabla r, \nabla r) \\
& +\frac{\alpha}{\alpha^{2}+(n-1) \alpha+4 K}(\widetilde{\Delta} r)^{2}+g(\nabla r, \nabla \widetilde{\Delta} r) .
\end{aligned}
$$

Applying the assumption (9) given in Theorem 2 to (44), it follows that

$$
0 \geq \partial_{r}(\widetilde{\Delta} r)+\frac{\alpha}{\alpha^{2}+(n-1) \alpha+4 K}(\widetilde{\Delta} r)^{2}+(n-1) H
$$

Because of $\widetilde{\Delta} r=\Delta r-g(\nabla \phi, \nabla r)+\frac{\alpha}{2 r}$, we have

$$
\begin{aligned}
\lim _{r \rightarrow 0^{+}} r \widetilde{\Delta} r & =\lim _{r \rightarrow 0^{+}}\left(r \Delta r-r g(\nabla \phi, \nabla r)+\frac{\alpha}{2}\right) \\
& =n-1+\frac{\alpha}{2} \leq \frac{\alpha^{2}+(n-1) \alpha+4 K}{\alpha} .
\end{aligned}
$$

Thus, with the aid of the well-known Sturm-Liouville comparison argument, we obtain

$$
\widetilde{\Delta} r \leq \sqrt{(n-1) H\left(n-1+\alpha+\frac{4 K}{\alpha}\right)} \cot \left(\frac{\sqrt{\alpha(n-1) H}}{\sqrt{\alpha^{2}+(n-1) \alpha+4 K}} r\right)
$$


on $M-\left(C_{p} \cup\{p\}\right)$. To conclude the proof of Theorem 2, we can use the same arguments given in [7]: Let $q \in M$ and let $\sigma$ be a minimizing unit speed geodesic segment from $p$ to $q$ where the point $p \in M$ is given in Theorem 2. Assume that

$$
d(p, q)>\frac{\sqrt{\alpha^{2}+(n-1) \alpha+4 K}}{\sqrt{\alpha(n-1) H}} \pi .
$$

Then, since $\sigma$ is a minimizing unit speed geodesic segment from $p \in M$ to $q \in M$, we have the fact

$$
\sigma\left(\frac{\sqrt{\alpha^{2}+(n-1) \alpha+4 K}}{\sqrt{\alpha(n-1) H}} \pi\right) \in M-\left(C_{p} \cup\{p\}\right) .
$$

Thus the distance function $r$ is smooth at this point. Namely, at this point, left hand side of (48) is a constant. But it is obvious that, when

$$
r \rightarrow\left(\frac{\sqrt{\alpha^{2}+(n-1) \alpha+4 K}}{\sqrt{\alpha(n-1) H}} \pi\right)^{-},
$$

right hand side of (48) goes to $-\infty$. This is a contradiction. Hence must be

$$
d(p, q) \leq \frac{\sqrt{\alpha^{2}+(n-1) \alpha+4 K}}{\sqrt{\alpha(n-1) H}} \pi .
$$

Here $\alpha=2 \sqrt{K}$ gives the minimum value of right hand side of (52). Inserting $\alpha=2 \sqrt{K}$ into (52), we find

$$
d(p, q) \leq \sqrt{4 \sqrt{K}+n-1} \frac{\pi}{\sqrt{(n-1) H}} .
$$

Thus, we have proved Theorem 2.

Open Access This article is distributed under the terms of the Creative Commons Attribution Noncommercial License which permits any noncommercial use, distribution, and reproduction in any medium, provided the original author(s) and source are credited.

\section{References}

1. Bakry, D., Émery, M.: Diffusions Hypercontractives. In: Séminaire de probabilitiés XIX, Lect. Notes in Math. 1123, 177-206 (1985)

2. Fernández-López, M., García-Río, E.: A remark on compact Ricci solitons. Math. Ann. 340, $893-896$ (2008)

3. Limoncu, M.: Modifications of the Ricci tensor and applications. Arch. Math. 95, 191-199 (2010)

4. Lott, J.: Some geometric properties of the Bakry-Émery-Ricci Tensor. Comment. Math. Helv. 78, 865-883 (2003)

5. Myers, S.B.: Riemannian manifolds with positive mean curvature. Duke Math. J. 8, 401-404 (1941)

6. Wei, G., Wylie, W.: Comparison geometry for the Bakry-Emery Ricci tensor. J. Differ. Geom. 83, 377-405 (2009)

7. Zhu, S.: The comparison geometry of Ricci curvature. Comparison Geometry. MSRI Publications, vol. 30, pp. 221-262 (1997) 